\title{
Stellar Populations in Star Clusters: The Rôle Played by Stochastic Effects
}

\author{
Gustavo Bruzual A. \\ Centro de Investigaciones de Astronomía (C.I.D.A.), Apartado Postal \\ 264, Mérida, Venezuela
}

\begin{abstract}
In this paper I combine the results of a set of population synthesis models with simple Montecarlo simulations of stochastic effects in the number of stars occupying sparsely populated stellar evolutionary phases, to show that the scatter observed in the magnitudes and colors of LMC and NGC 7252 star clusters can be understood in the framework of current stellar evolution theory, without the need to introduce ad-hoc corrections (e.g. artificially increasing the number of AGB stars).
\end{abstract}

\section{Introduction}

When photometric properties of star clusters are compared to the predictions of population synthesis models, it is common to find that the scatter among the observed colors and the difference between these colors and the model predictions, are larger than allowed by the observational errors. This is particularly true for intermediate age clusters in optical-IR colors, like $V-K$, but to a lesser extent the scatter is also large in $B-V$. Fig 1 illustrates this point.

In a recent paper, Maraston et al. (2001) show that the population synthesis models of Maraston (1998) reproduce quite well the $V-K$ colors of the cluster population observed in NGC 7252 at an age between 400 and 600 Myr. In these models, built on the fuel consumption theorem (Renzini \& Buzzoni 1986), the contribution of AGB stars to the bolometric light in the synthetic population has been increased over that expected from stellar evolution theory, in such a way as to reproduce the empirical values determined by Frogel et al. (1990) for a sample of LMC clusters as a function of SWB class. Even though this is a valid procedure, in this paper I show that the optical and optical-IR colors of the intermediate age clusters of the LMC and NGC 7252 are consistent with the colors expected from models (Bruzual \& Charlot 2001; Bruzual 2000, 2001) based on current stellar evolution theory, if stochastic fluctuations in the number of stars in sparsely populated evolutionary stages are properly included into the models. In what follows I use the Montecarlo technique pioneered by Barbaro \& Bertelli (1977), Chiosi et al. (1988), Girardi et al. (1995), and Santos \& Frogel (1997), to study the role that stochastic effects on the initial mass function (IMF) have on star cluster colors following the Santos \& Frogel (1997) approach, and apply it to the LMC and NGC 7252 cluster samples. 
Padova (1994), Salpeter IMF
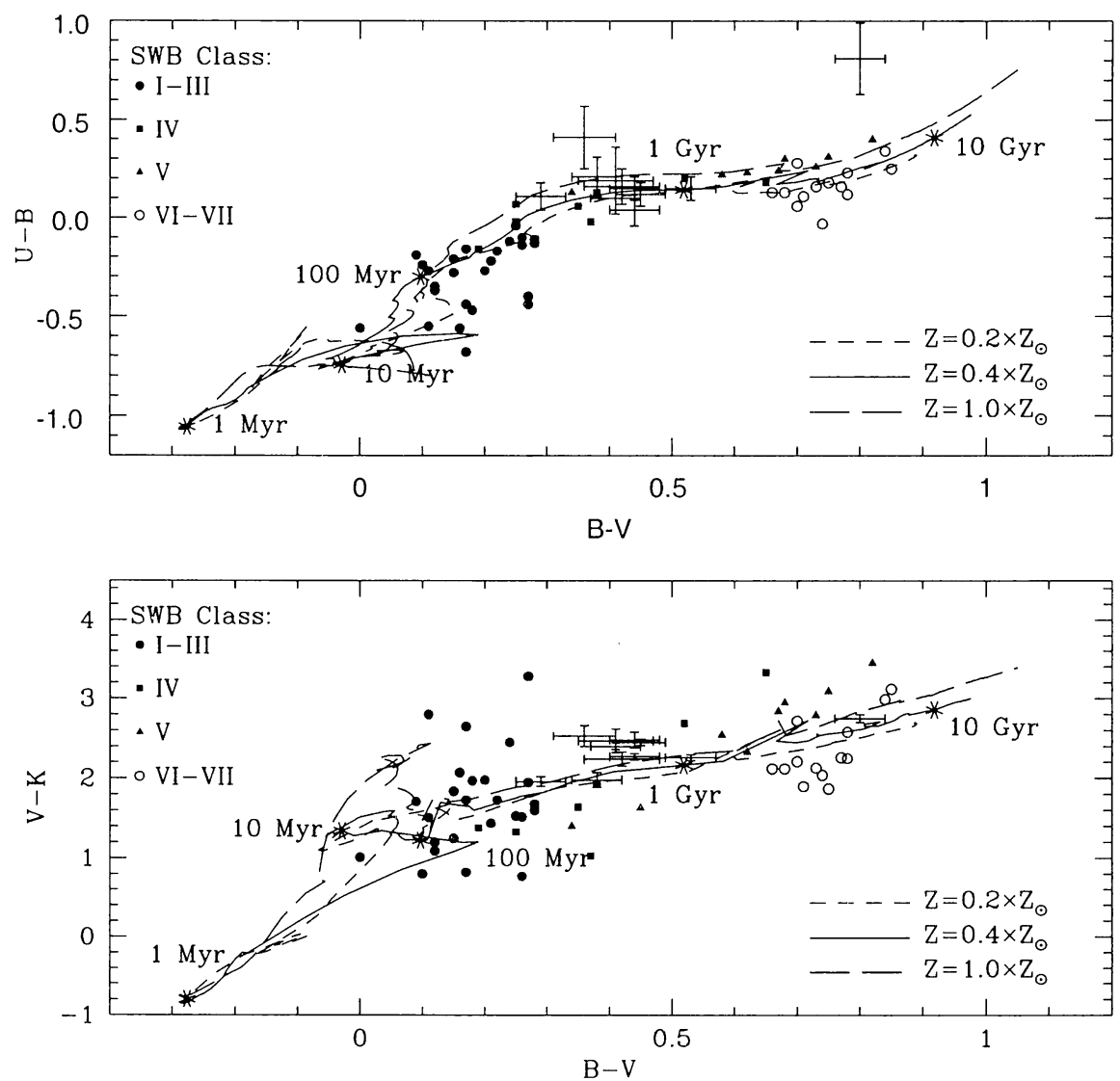

Figure 1. $\quad U-B$ vs. $B-V$ and $V-K$ vs. $B-V$ color diagrams. The solid dots, squares, triangles, and open circles represent LMC globular clusters discriminated according to their SWB class, as indicated in the upper left corner of each frame. $B-V$ comes from van den Bergh (1981) and $V-K$ from Persson et al. (1983). The points with error bars correspond to the star clusters in NGC 7252 from Maraston et al. (2001). The lines represent the evolution in this plane of BC2000 SSP models (Bruzual 2000) computed using the Padova (Fagotto et al. 1994a,b,c) evolutionary tracks for $Z=0.2 \times Z_{\odot}, 0.4 \times Z_{\odot}$, and $Z_{\odot}$, the Salpeter (1955) IMF, and the Lejeune et al. (1998) stellar atlas. The * symbols along the $Z=0.4 \times Z_{\odot}$ line mark the model colors at the age indicated by the labels, and can be used to roughly date the clusters. 


\section{Method}

The derivation below follows closely Santos \& Frogel (1997). The IMF

$$
\Phi(m)=d N / d m=C m^{-(1+x)},
$$

normalized as usual,

$$
C=\frac{x}{m_{l}^{-x}-m_{u}^{-x}}
$$

obeys, $\Phi(m) \geq 0$, and

$$
\int_{m_{l}}^{m_{u}} \Phi\left(m^{\prime}\right) d m^{\prime}=1 .
$$

$\Phi(m)$ can be interpreted as a probability distribution function which gives the probability that a random mass $m^{\prime}$ is in the range between $m$ and $m+d m$. $\Phi(m)$ can be transformed into another probability distribution function $g(N)$, such that the probability of occurrence of the random variable $N^{\prime}$ within $d N$ and the probability of occurrence of the random variable $m^{\prime}$ within $d m$ is the same,

$$
|\Phi(m) d m|=|g(N) d N| .
$$

where $N$ is a single-valued function $m$. From (1),

$$
N(m)=\int_{m_{l}}^{m} \Phi\left(m^{\prime}\right) d m^{\prime},
$$

is a cumulative distribution function which gives the probability that the mass $m^{\prime}$ is less or equal to $m$, and using (4), it follows that

$$
g(N)=1, \quad 0 \leq N \leq 1 .
$$

$g(N)$ is thus a uniform distribution for which any value is equally likely in the interval $0 \leq N \leq 1$. If we sample $N$ using a random number generator (Press et al. 1992), from (5) we can obtain $m$ as a function of $N$,

$$
m=\left[(1-N) m_{l}^{-x}+N m_{u}^{-x}\right]^{-\frac{1}{x}} .
$$

For each star of mass $m$ generated in this way, we obtain its observational properties from the $\log T_{\text {eff }}$ and $\log L$ corresponding to this mass in the isochrone at the age of interest. We repeat the procedure until the cluster mass, i.e. the sum of $m$ for all the stars generated, including dead stars, reaches the desired value. Adding the contribution of each star to the flux in different photometric bands, we obtain the cluster magnitude and colors in these bands. Throughout this paper I use $x=1.35$ (Salpeter 1955), and for the lower and upper mass limits of star formation, I assume $m_{l}=0.09$ and $m_{u}=125 \mathrm{M}_{\odot}$, respectively. 
Tracks: Padova (1994), Z=0.008, Salpeter IMF

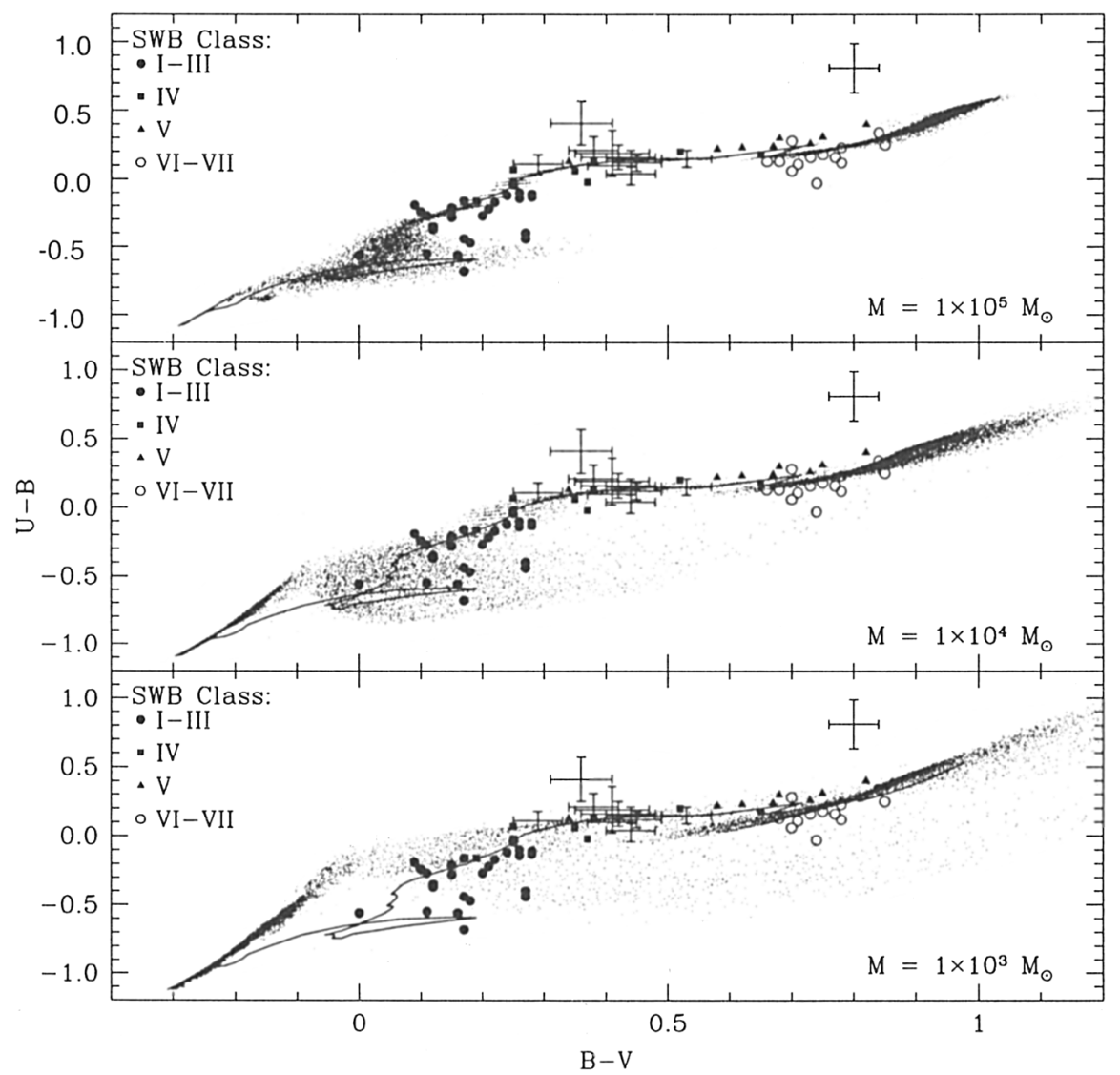

Figure 2. The small dots indicate the $U-B$ and $B-V$ colors resulting from different simulations in which stochastic fluctuations in the number of stars that populate different evolutionary stages are taken into account as indicated in the text. 100 simulations were run at each of 220 time steps or isochrones, obtained from the BC2000 SSP model (Bruzual 2000) for the Padova (Fagotto et al. 1994a,b,c) tracks for $Z=0.4 \times Z_{\odot}$, the Salpeter (1955) IMF, and the Lejeune et al. (1998) stellar atlas (uncorrected atmospheres). The simulation is stopped when the total cluster mass (including dead stars) reaches $1 \times 10^{5}, 1 \times 10^{4}$, and $1 \times 10^{3} \mathrm{M}_{\odot}$ (top to bottom), as indicated in the lower right corner of each frame. The fluctuations in the colors become larger as the cluster mass decreases. The number of stars in the $1 \times 10^{3}$ $\mathrm{M}_{\odot}$ cluster may be unrealistically small, and some evolutionary stages are not sampled. The data points and the solid line for $Z=0.4 \times Z_{\odot}$ are the same as in Fig 1. The solid line represents the evolution of this model with no fluctuations, which is equivalent to an infinite number of stars populating the IMF. 
Tracks: Padova (1994), $\mathrm{Z}=0.008$, Salpeter IMF

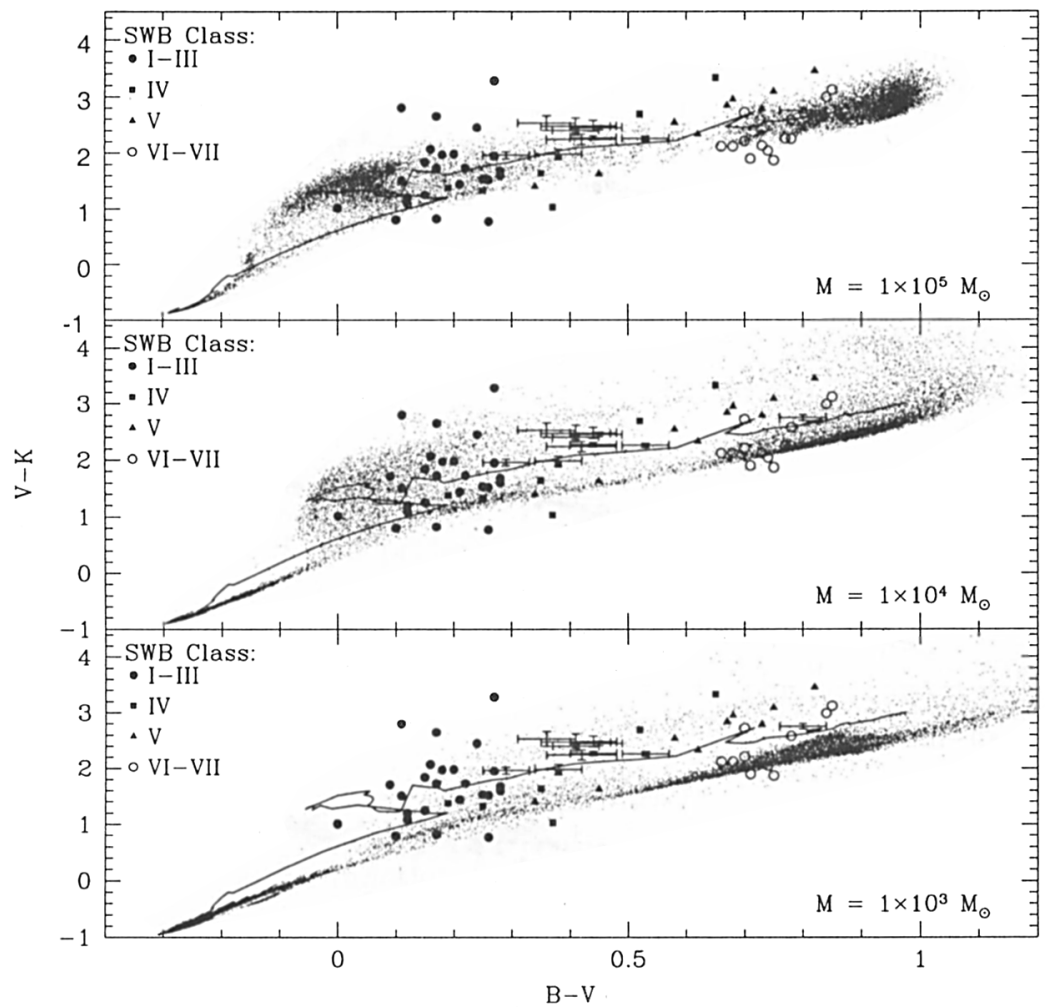

Figure 3. The small dots indicate the $V-K$ and $B-V$ colors resulting from different simulations in which stochastic fluctuations in the number of stars that populate different evolutionary stages are taken into account as indicated in the text. 100 simulations were run at each of 220 time steps or isochrones, obtained from the BC2000 SSP model (Bruzual 2000) for the Padova (Fagotto et al. 1994a,b,c) tracks for $Z=0.4 \times Z_{\odot}$, the Salpeter (1955) IMF, and the Lejeune et al. (1998) stellar atlas (uncorrected atmospheres). The simulation is stopped when the total cluster mass (including dead stars) reaches $1 \times 10^{5}, 1 \times 10^{4}$, and $1 \times 10^{3} \mathrm{M}_{\odot}$ (top to bottom), as indicated in the lower right corner of each frame. The fluctuations in the colors become larger as the cluster mass decreases. The number of stars in the $1 \times 10^{3}$ $\mathrm{M}_{\odot}$ cluster may be unrealistically small, and some evolutionary stages are not sampled. The data points and the solid line for $Z=0.4 \times Z_{\odot}$ are the same as in Fig 1. The solid line represents the evolution of this model with no fluctuations, which is equivalent to an infinite number of stars populating the IMF. The expected fluctuations in $V-K$ for a $1 \times 10^{4} \mathrm{M}_{\odot}$ cluster amount to almost 2 magnitudes, in close agreement with the range of colors observed at a given age. 


\section{Results}

Figs 2 to 5 show the results of the Montecarlo simulations. For reasons of space the details of each figure given in the caption are not repeated in the text. From Figs 2 and 3 it is apparent that the fluctuations in the colors become larger as the cluster mass decreases. The number of stars in the $1 \times 10^{3} \mathrm{M}_{\odot}$ cluster may be unrealistically small, and some evolutionary stages are not sampled. There are not enough high mass MS stars in the $1 \times 10^{3} \mathrm{M}_{\odot}$ and $1 \times 10^{4} \mathrm{M}_{\odot}$ clusters to make them as blue in $U-B$ at early ages as the model computed with the analytical IMF (equivalent to infinite number of stars). In the $1 \times 10^{5} \mathrm{M}_{\odot}$ case the upper MS is well sampled and both models are equally blue in $U-B$. Fig 3 shows that the lower mass cluster models at early ages are bluer in $V-K$ than the analytic model because the lower MS is over populated with respect to the short lived phases which redden this color. For the $1 \times 10^{5} \mathrm{M}_{\odot}$ cluster the agreement is good at early ages. The expected fluctuations in $V-K$ for a $1 \times 10^{4} \mathrm{M}_{\odot}$ cluster amount to almost 2 magnitudes, in close agreement with the range of colors observed at a given age, and is considerably broader than for the $1 \times 10^{4} \mathrm{M}_{\odot}$ case. Fig 4 shows an enlargement of the diagrams for the $1 \times 10^{4} \mathrm{M}_{\odot}$ star cluster. At the intermediate ages the models are redder in $V-K$ than the analytic IMF model because of a larger number of AGB stars, which appear naturally as a consequence of stochastic fluctuations in the IMF. The regions in these planes occupied by the observations and the cluster simulations overlap quite well. This comparison seems to favor a not very large mass for these clusters, if stochastic fluctuations are responsible for the color variations. This conclusion is supported by Fig 5 in which I plot $K$ vs $V-K$ at the distance of the LMC. The region covered by the $1 \times 10^{4} \mathrm{M}_{\odot}$ clusters matches the region covered by the observations very well. This is not the case for the $1 \times 10^{5} \mathrm{M}_{\odot}$ clusters, which show a much lower dispersion in color for the same $K$ magnitude than the observations and the lower mass simulations.

\section{Conclusions}

Even though the Montecarlo technique used in this paper has been used successfully before to explain cluster colors, I have revisited it in this paper to show that there is no need to introduce ad-hoc assumptions into population synthesis models (Maraston 1998, Maraston et a. 2001), which represent a departure from our current understanding of stellar evolution theory, in order to explain the observed range of values of cluster colors and magnitudes. In this paper I have briefly summarized the results of simple simulations which show clearly how the range of colors observed in intermediate age star clusters can be understood on the basis of current stellar evolution theory, if we take into account properly the expected variation in the number of stars occupying sparsely populated evolutionary stages due to stochastic fluctuations in the IMF.

Our preliminary conclusion that cluster masses in the range around $1 \times 10^{4}$ $\mathrm{M}_{\odot}$ are preferred, as well as the dependence on this mass of the fluctuations in magnitude and colors will be explored in detail by Bruzual \& Charlot (2001). 
Padova (1994), $\mathrm{Z}=0.008$, Salpeter IMF
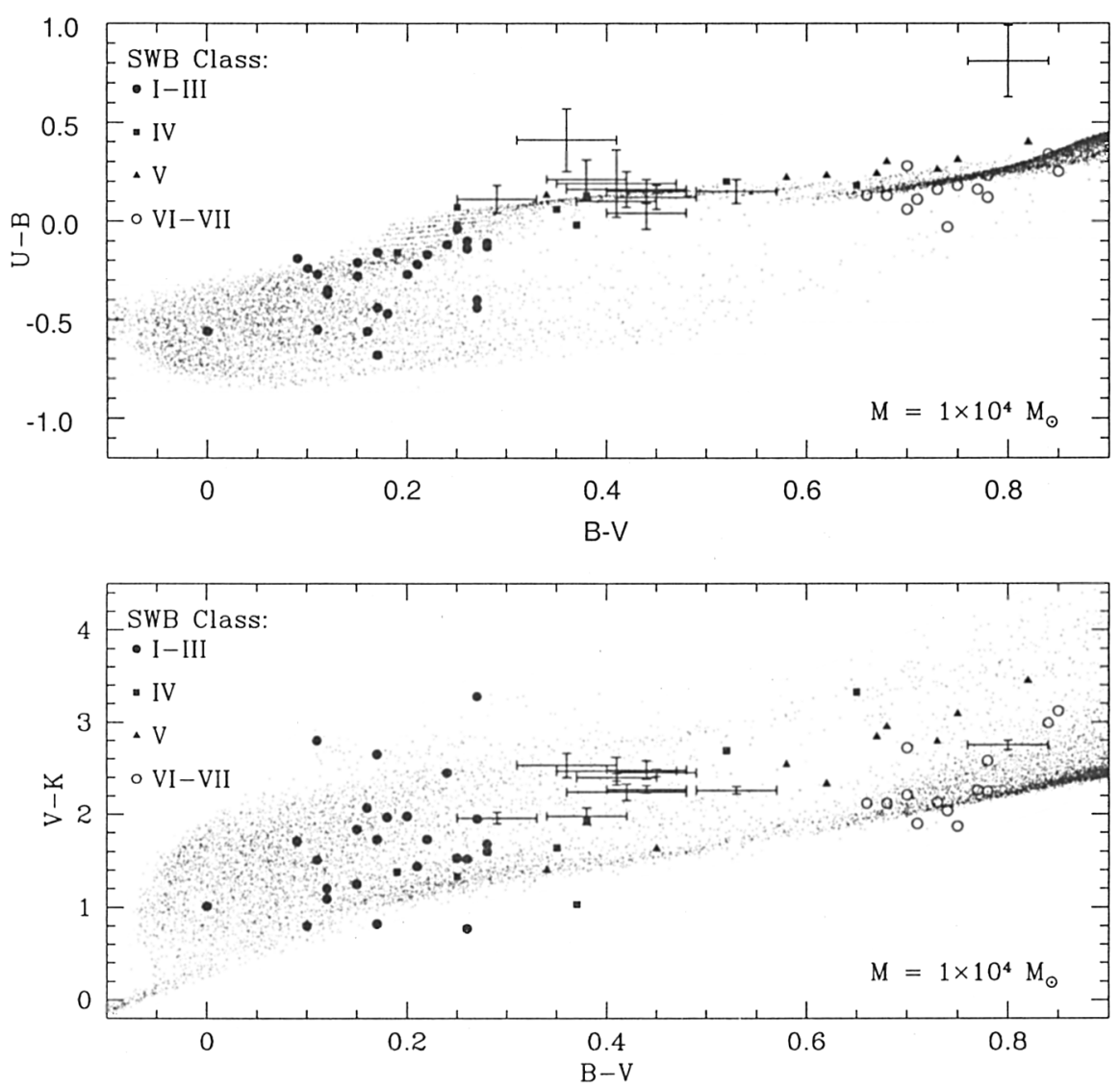

Figure 4. Enlarged view of the $U-B$ vs. $B-V$ and $V-K$ vs. $B-V$ color diagrams shown in the middle frame of Figs 2 and 3. The data points are the same as in Fig 1 . The regions in these planes occupied by the observations and the cluster simulations overlap very well. 
Padova (1994), Z=0.008, Salpeter IMF
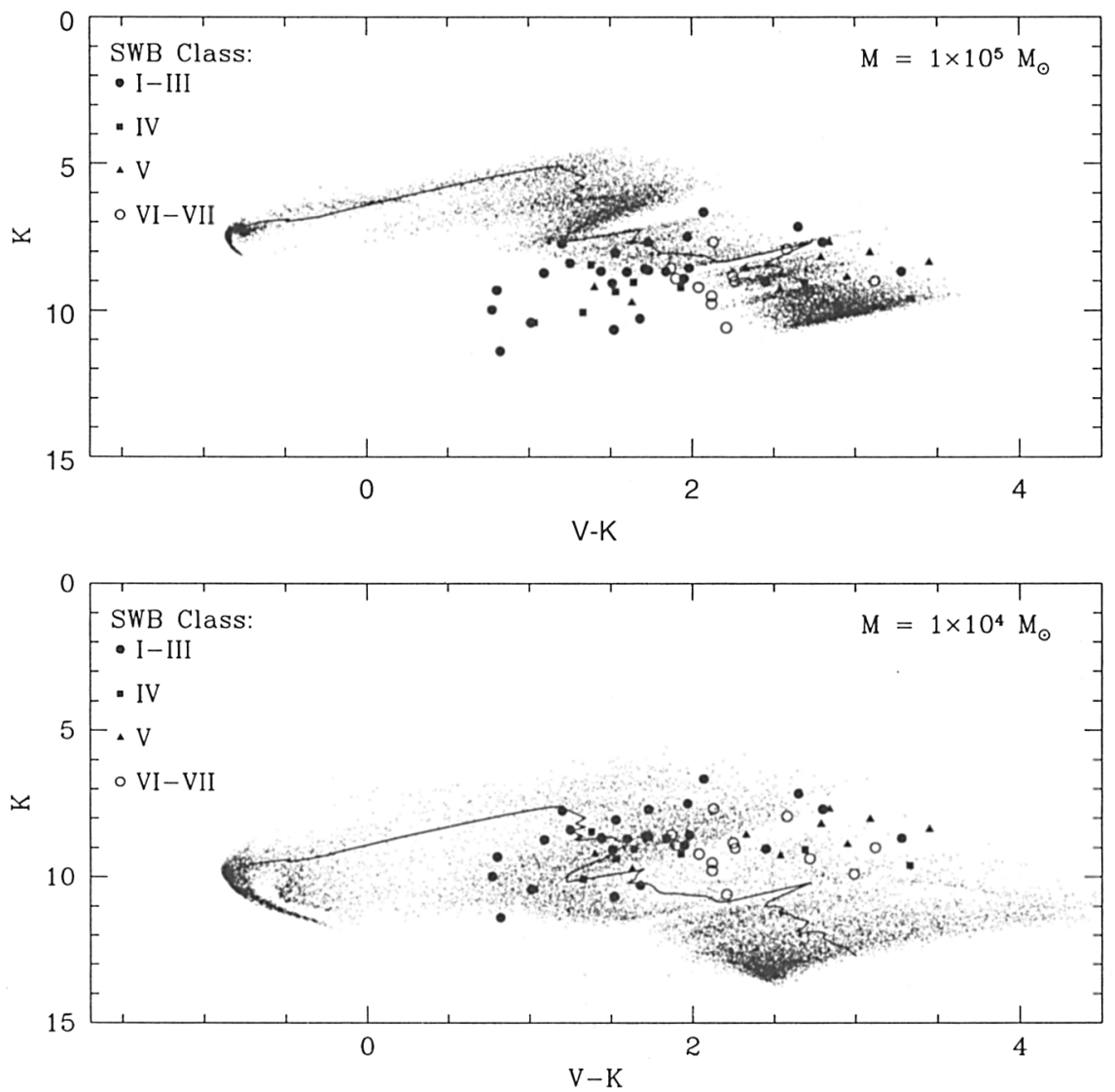

Figure 5. The small dots indicate the $K$ magnitude (at the distance of the LMC) and $V-K$ color resulting from different simulations in which stochastic fluctuations in the number of stars that populate different evolutionary stages are taken into account as indicated in the text. 100 simulations were run at each of 220 time steps or isochrones, obtained from the BC2000 SSP model (Bruzual 2000) for the Padova (Fagotto et al. 1994a,b,c) tracks for $Z=0.4 \times Z_{\odot}$, the Salpeter (1955) IMF, and the Lejeune et al. (1998) stellar atlas (uncorrected atmospheres). The simulation is stopped when the total cluster mass (including dead stars) reaches $1 \times 10^{5}$ (top) and $1 \times 10^{4} \mathrm{M}_{\odot}$ (bottom), as indicated in the upper right corner of each frame. The fluctuations in the colors get considerably larger as the cluster mass decreases. The data points are the same as in Fig 1 . The solid line represents the evolution of this model with no fluctuations, which is equivalent to an infinite number of stars populating the IMF. 
The interested reader may request results for other quantities equally sensitive to stochastic fluctuations, like the number of ionizing photons from a young stellar population, not shown here for lack of space.

\section{References}

Barbaro, G. \& Bertelli, G. 1977, A\&A, 54,243

Bruzual A., G. 2000, in Proc. of XI Canary Islands Winter School of Astrophysics on "Galaxies at High Redshift", eds. I. Pérez-Fournon and F. Sánchez, Cambridge Cont. Astrophys., in press, (BC2000, astro-ph/0011094)

Bruzual A., G. 2001 in Proc. of Euroconf. "The Evolution of Galaxies. I Observational Clues",eds. G. Stasińska \& J. Vílchez, Dordrecht: Kluwer, in press

Bruzual A., G. \& Charlot, S. 2001, in preparation

Chiosi, C., Bertelli, G., \& Bressan, A. 1988, A\&A, 196, 84

Fagotto, F., Bressan, A., Bertelli, G., \& Chiosi, C. 1994a, A\&AS, 100, 647

Fagotto, F., Bressan, A., Bertelli, G., \& Chiosi, C. 1994b, A\&AS, 104, 365

Fagotto, F., Bressan, A., Bertelli, G., \& Chiosi, C. 1994c, A\&AS, 105, 29

Frogel, J.A., Mould, J., \& Blanco, V.M. 1990, ApJ, 352, 96

Girardi, L., Chiosi, C., Bertelli, G., \& Bressan, A. 1995, A\&A, 298, 87

Lejeune, T., Cuisinier, F., \& Buser, R. 1998, A\&AS, 130, 65

Maraston, C. 1998, MNRAS, 300, 872

Maraston, C., Kissler-Patig, M., Brodie, J.P., Barmby, P., \& Huchra, J.P. 2001, A\&A, 370, 176

Persson, S.E., Aaronson, M., Cohen, J.G., Frogel, J.A. \& Mathews, K. 1983, ApJ, 266, 105

Press, W.H., Teukolsky, S.A., Vetterling, W.T., \& Flannery, B.P. 1992, Numerical Recipes in FORTRAN, 2nd ed., Cambridge: Cambridge Univ. Press

Renzini, A., \& Buzzoni, A. 1986, in "Spectral Evolution of Galaxies", eds. C. Chiosi and A. Renzini, Dordrecht: Reidel, p. 195

Salpeter, E.E. 1955, ApJ, 121, 161

Santos, Jr, J.F.C. \& Frogel, J.A. 1997, ApJ, 479, 764

van den Bergh, S. 1981, A\&AS, 46, 79

\section{Discussion}

F. D'Antona: I would like to note the twofold role of stellar evolution people in providing new models. They must emphasize what is very parametric and must not be overtrusted. But on the other hand, there are real improvements made in the models from time to time. E.g., inclusion of rotation, as made in the latest Geneva tracks, will certainly alter the picture at "short" ages. These improvements are important, even if they worsen agreement with observations, as only a disagreement can push our understanding forward, as demonstrated by the "paradigm" of the ages of globular clusters in recent years. 